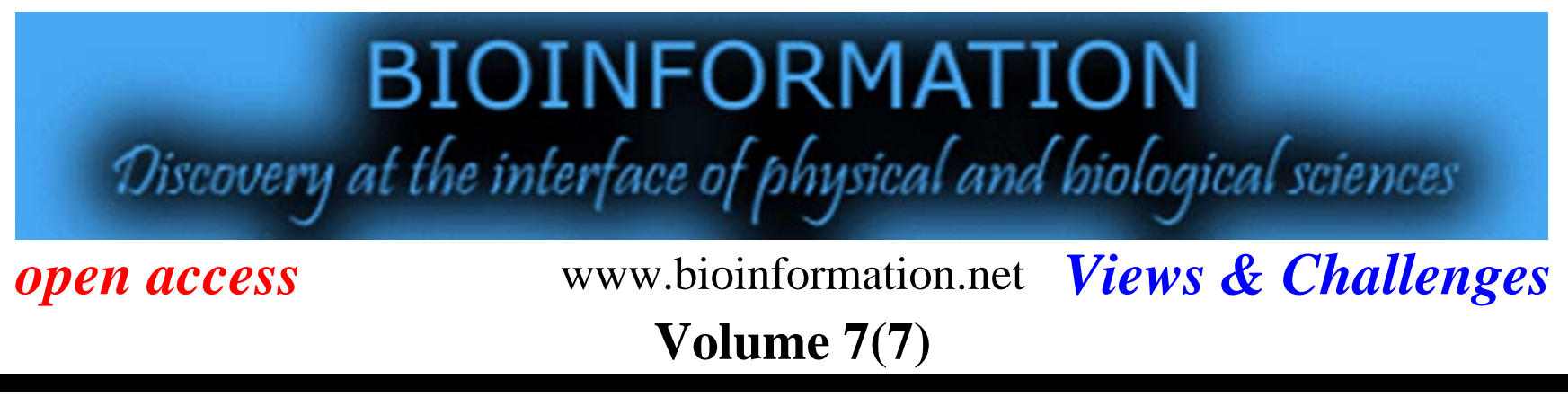

\title{
Monozygotic twins: genes are not the destiny?
}

\author{
Aniruddha Chatterjee ${ }^{1,2}$, Ian M Morison"1, 2
}

1Department of Pathology, Dunedin School of Medicine, University of Otago, 270 Great King Street, Dunedin 9054, New Zealand; ${ }^{2}$ National Research Centre for Growth and Development, New Zealand; Aniruddha Chatterjee - Email: aniruddha.chatterjee@otago.ac.nz; Phone: +64-3-470-3455; *Corresponding author

Received December1, 2011; Accepted December 5, 2011; Published December 10, 2011

\begin{abstract}
:
Monozygotic twins are considered to be genetically identical, yet can show high discordance in their phenotypes and disease susceptibility. Several studies have emphasized the influence of external factors and the role of epigenetic polymorphism in conferring this variability. However, some recent high-resolution studies on DNA methylation show contradicting evidence, which poses questions on the extent of epigenetic variability between twins. The advent of next-generation sequencing technologies now allow us to interrogate multiple epigenomes on a massive scale and understand the role of epigenetic modification, especially DNA methylation, in regulating complex traits. This article briefly discusses the recent key findings, unsolved questions in the area, and speculates on the future directions in the field
\end{abstract}

Keywords: monozygotic twins, epigenetics, DNA methylation, next-generation sequencing

\section{Background:}

Monozygotic (MZ) twins originate when a single egg is fertilized to form one zygote, which then divides into two embryos. Although they share the same genotype they are not phenotypically identical. Within monozygotic twin pairs there can be significant discordance for some multi-factorial diseases $[1,2]$ and they also show a wide range of anthropomorphic features. However, the nature, source and occurrence of discordance are poorly understood. Epigenetic modification is a plausible mechanism to explain twin discordance than genetic or stochastic mechanisms alone.

\section{Untangling the epigenetic potential:}

The first large-scale study of DNA methylation on twins compared 20 pairs each of MZ and dizygotic (DZ) twins and showed similar epigenetic profiles in MZ twins, indicating high heritability. Epigenetic variation, however, accumulated with increasing age [3]. Since it was not a longitudinal study it did not allow comparison of epigenetic profiles in the same individuals over time. Continuing on this line several other studies provided strong evidence in favor of epigenetic heritability and an effect of age and other non-genetic factors in inducing epigenetic variation in $\mathrm{MZ}$ twins during their life time $[4,5]$. High resolution DNA methylation assays revealed a large degree of variation in different tissues and identified almost 6000 unique metastable genomic regions in MZ twins [5], indicative of extensive tissue-specific variation in $\mathrm{MZ}$ twins.

In another study, epigenetic analysis of different tissues from newborn MZ twins showed considerable variation in DNA methylation and the presence of methylation discordance within twin pairs, suggesting the importance of the maternal environment in forming the epigenome of a newborn and the sensitivity of the intrauterine period to induce epigenetic variation [6]. A longitudinal study on $M Z$ twins showed differences in DNA methylation pattern in childhood that were not stable over their lifetime and the authors concluded that environmental influences are responsible for the changes in DNA methylation and they were not heritable [7]. A more recent study using bisulphite sequencing, supported the notion of low heritability and significant variation in DNA methylation in $\mathrm{MZ}$ twins [8]. Epigenetic differences provide a plausible explanation for disease discordance between $\mathrm{MZ}$ twins and tempts to speculate on disease risks of individual epigenetic 
effects [9]. Several studies have reported profound phenotypeassociated DNA methylation differences between MZ discordant twin pairs in human diseases [1, 10, 11]. DNA methylation at the imprinted KCNQ1OT1 region accounts for discordance for Beckwith-Wiedemann syndrome in MZ twins [12]. For systemic lupus erythematosus MZ twins displayed significant changes in the DNA methylation status (49 differentially methylated regions were identified) of immunesystem-related genes [13]. In addition, a recent study found disease-associated differences in DNA methylation in MZ twins discordant for schizophrenia and bipolar disorders [14].

In contrast, a study that used next generation sequencing (NGS) for the first time in MZ twins identified a very low level of differences in DNA methylation. CD4+ lymphocyte DNA from three monozygotic twin pairs that were discordant for multiple sclerosis were sequenced and only 2, 10 and 176 differentially methylated sites were identified out of 2 million CpG dinucleotides interrogated within the three twin pairs [15]. One criticism could be that only three pairs of twins were analyzed and they were heterogeneous mix of males, females and different ethnicity. Nevertheless, the study provides highresolution information on DNA methylation and contradicts with the previous findings that epigenetic differences are the major reason for disease discordance in $\mathrm{MZ}$ twins.

\section{Perspective and speculation:}

As presented, the literature provides contradicting evidence and leaves us with some questions. Firstly, at what stage does epigenetic variation arise in MZ twins. Secondly, to what extent do non-genetic factor influence the changes. Thirdly, do epigenetic changes confer phenotypic variation and heritability in twins. Finally, what is the role of DNA methylation change in disease discordant twins.

Studies to date have broadened our understanding of epigenetic phenomena in MZ twins, but the majority of the studies are of low resolution and covered a small fraction of CpG sites. The rapid improvement in NGS technologies are promising and are capable of resolving DNA methylation status at single base pair resolution at high genomic coverage and in multiple samples. Several excellent tools that harness the power of NGS have been developed recently, such as RRBS (reduced representation bisulphite sequencing) [16], MeCAP-seq (methylated DNA capture by affinity purification sequencing) [17], and MeDIP-seq (methylated DNA immunoprecipitation sequencing) [18], allowing multiple epigenomes of twins to be analysed. The advent of single-molecule sequencing has potential to reveal more epigenetic modifications and provide deeper insight into complex phenotypic traits in $\mathrm{MZ}$ twins.
To obtain a conclusive answer about the role of epigenetics in twin discordance, future studies will need to be well designed to consider tissue heterogeneity, the contribution of environmental factors, and longitudinal stability. It is promising that several large-scale twin studies, which apply latest technologies to unravel epigenetic marks, are well underway. MuTHER (http://www.muther.ac.uk/), EpiTwin (http://www.twinsuk.ac.uk/projects/epitwin.html), and ENGAGE (http://www.euengage.org/) consortium are some examples. As MZ twins share common DNA sequence, they offer an excellent opportunity to understand the mechanism of complex traits and disease etiology from an epigenetic perspective, which is beyond the explanation of Mendelian genetics.

\section{Acknowledgements:}

We acknowledge the support of National Research Centre for Growth and Development, New Zealand. We are thankful to Dr. Euan Rodger for his support and valuable inputs on the manuscript. We thank Dr. Peter Stockwell for his feedback on the manuscript.

\section{References:}

[1] Petronis A et al. Schizophr Bull. 2003 29: 169 [PMID: 12908672]

[2] Wong $\mathrm{AH}$ et al. Hum Mol Genet. 200514 Spec No 1: R11 [PMID: 15809262]

[3] Fraga MF et al. Proc Natl Acad Sci U S A. 2005 102: 10604 [PMID: 16009939]

[4] Heijmans BT et al. Hum Mol Genet. 2007 16: 547 [PMID: 17339271]

[5] Kaminsky ZA et al. Nat Genet. 2009 41: 240 [PMID: 19151718]

[6] Ollikainen M et al. Hum Mol Genet. 2010 19: 4176 [PMID: 20699328]

[7] Wong CC et al. Epigenetics. 2010 5: 516 [PMID: 20505345]

[8] Gervin K et al. Genome Res. 2011 21: 1813 [PMID: 21948560]

[9] Bell JT \& Spector TD, Trends Genet. 2011 27: 116 [PMID: 21257220]

[10] Kuratomi G et al. Mol Psychiatry. 2008 13: 429 [PMID: 17471289]

[11] Oates NA et al. Am J Hum Genet. 2006 79: 155 [PMID: 16773576]

[12] Weksberg R et al. Hum Mol Genet. 200312 Spec No 1: R61 [PMID: 12668598]

[13] Javierre BM et al. Genome Res. 2010 20: 170 [PMID: 20028698]

[14] Dempster EL et al. Hum Mol Genet. 2011 20: 4786 [PMID: 21908516]

[15] Baranzini SE et al. Nature. 2010 464: 1351 [PMID: 20428171]

[16] Meissner A et al. Nature. 2008 454: 766 [PMID: 18600261]

[17] Brinkman AB et al. Methods. 2010 52: 232 [PMID: 20542119]

[18] Weber M et al. Nat Genet. 2005 37: 853 [PMID: 16007088] 